\title{
The clinical utility of tumor mutational burden in non-small cell lung cancer
}

\author{
Laurent Greillier ${ }^{1,2}$, Pascale Tomasini ${ }^{1,2}$, Fabrice Barlesi ${ }^{1,2}$ \\ ${ }^{1}$ Aix Marseille University, Assistance Publique Hôpitaux de Marseille, Multidisciplinary Oncology \& Therapeutic Innovations department, Marseille, \\ France; ${ }^{2}$ Predictive Oncology Laboratory, Centre de Recherche en Cancérologie de Marseille, Inserm UMR1068, CNRS UMR7258, Aix-Marseille \\ Université UM105, Marseille, France \\ Contributions: (I) Conception and design: All authors; (II) Administrative support: All authors; (III) Provision of study materials or patients: All \\ authors; (IV) Collection and assembly of data: All authors; (V) Data analysis and interpretation: All authors; (VI) Manuscript writing: All authors; (VII) \\ Final approval of manuscript: All authors. \\ Correspondence to: Prof. Fabrice Barlesi. Service d’Oncologie Multidisciplinaire et Innovations Thérapeutiques, Hôpital Nord, Chemin des Bourrely, \\ 13915 Marseille Cedex, France. Email: fabrice.barlesi@ap-hm.fr.
}

\begin{abstract}
Despite advances made during the last two decades, lung cancer remains the leading cause of cancer-related death worldwide. Recently, immune checkpoint inhibitors (ICIs) became available for the treatment of advanced non-small cell lung cancer (NSCLC) patients. Although ICIs showed a survival advantage in comparison with chemotherapy in the second and first-line setting, overall response rate is only around $20 \%$ and a large proportion of patients will undergo disease progression within the first weeks of treatment. For this reason, there is a need for biomarkers to predict the efficacy of ICIs in NSCLC and to identify patients who will benefit from ICIs. The first biomarker developed was programmed cell death ligand 1 (PD-L1) expression. However, the predictive role of PD-L1 expression varied from one clinical trial to another, due to the multiple assays used, with different antibodies, different platforms, and different thresholds. Moreover, PD-L1 expression is highly heterogeneous. For these reasons, PD-L1 expression alone is not a good biomarker to predict the efficacy of ICIs and there is a need for the identification of other biomarkers. Tumor mutational burden (TMB) is defined as the number of mutations per DNA megabases. It was first assessed as a biomarker for ICI based on the observation of successful immune checkpoint inhibition in solid tumors with high TMB such as NSCLC, melanoma or bladder cancer. Pre-clinical data suggested that the association between TMB and ICIs efficacy could be explained by the creation of neoantigens induced by mutations acquisition, increasing tumor immunogenicity and response to ICIs. Preliminary observations of TMB role as a predictive biomarker for the efficacy of ICIs in patients with advanced NSCLC led to the assessment of TMB clinical utility in phase III clinical trials. This review reports the clinical features and prognostic role of TMB in NSCLC. This review also focuses on TMB predictive role for the treatment of NSCLC patients with single-agent programmed cell death 1 (PD-1) and PD-L1 inhibitors such as pembrolizumab, nivolumab and atezolizumab or ICIs combination.
\end{abstract}

Keywords: Tumor mutational burden (TMB); immune checkpoint inhibitors (ICIs); non-small cell lung cancer (NSCLC)

Submitted Aug 01, 2018. Accepted for publication Oct 10, 2018.

doi: $10.21037 /$ tlcr.2018.10.08

View this article at: http://dx.doi.org/10.21037/tlcr.2018.10.08 


\section{Introduction}

Lung cancer is the most often diagnosed cancer in men and continues to be the main source of cancer-related deaths around the world, with more than 1 million deaths in 2012 (1). During the last two decades, a better knowledge of the biology of advanced non-small cell lung cancer (NSCLC) led to the identification of predictive biomarkers such as EGFR and BRAF mutations or ALK and ROS1 rearrangements and to the development of matched targeted therapies. These discoveries improved the outcomes of patients with advanced NSCLC and a targetable molecular alteration (2-8). However, a targetable molecular alteration is found in only half cases of non-squamous NSCLC (9) and progress still has to be made to improve the clinical outcomes of the other half of patients with NSCLC.

More recently, immune checkpoint inhibitors (ICIs) became available for the treatment of advanced NSCLC patients. The programmed cell death 1 (PD-1) inhibitors nivolumab and pembrolizumab and the programmed cell death ligand 1 (PD-L1) inhibitor atezolizumab showed a survival advantage over docetaxel for the second line treatment of advanced NSCLC (10-13). Whereas nivolumab and atezolizumab efficacy was independent from PD-L1 expression, pembrolizumab Keynote 010 trial only enrolled PD-L1 positive ( $\geq 1 \%)$ NSCLC patients. In addition, pembrolizumab was associated with a longer OS in comparison with chemotherapy in the first-line setting of patients with advanced NSCLC harboring a PD-L1 expression $\geq 50 \%$ tumor cells (14). Finally, the PD-L1 inhibitor durvalumab was responsible for a longer PFS in comparison with placebo after chemo-radiation for the treatment of stage III NSCLC (15).

However, although these phase III studies showed a survival improvement with ICIs in comparison with standard of care and led to ICIs approval for the treatment of NSCLC patients, overall response rate with ICIs is only around $20 \%$ and a large proportion of patients will undergo disease progression within the first weeks of treatment. For this reason, there is a need for biomarkers to predict the efficacy of ICIs in NSCLC and to identify patients who will benefit from ICIs. The characteristics of cancerimmune system interaction have been studied to identify potential biomarkers (16). Among potential biomarkers are MHC expression, IFN sensitivity, lymphocyte count (including effector and regulatory $\mathrm{T}$ cells count), tumor T cells, IL-6 expression, PD-L1 expression or tumor mutational burden (TMB) (17). PD-L1 expression is the only biomarker available. In most phase III trials of PD-1 and PD-L1 inhibitors PD-L1 expression predictive role was assessed (18). Although it showed promising results, it's utility as a biomarker varied from one clinical trial to another and PD-L1 expression is only required for pembrolizumab while nivolumab, durvalumab or atezolizumab can be prescribed regardless of PD-L1 expression. This variability can be explained by the multiple assays used in clinical trials, with different antibodies, different platforms, and different thresholds. Moreover, PD-L1 expression is highly heterogeneous, with a low inter-observer and inter-assay reproducibility (19). Whereas efforts have been made to standardize PD-L1 assays and decrease their variability (20), PD-L1 expression alone is not a good biomarker to predict ICIs efficacy. For these reasons, there is a need for the identification of other biomarkers.

TMB is defined as the number of mutations per DNA megabases $(\mathrm{Mb})$. TMB was assessed as a predictive biomarker for ICIs efficacy based on the observation of successful immune checkpoint inhibition in solid tumors with high TMB such as NSCLC, melanoma or bladder cancer (21). Rizvi et al. showed an association between high TMB and durable clinical benefit from ICIs in a population of advanced NSCLC patients (22). This could be explained by the creation of neoantigens induced by mutations acquisition, increasing tumor immunogenicity and response to ICIs (23).

This review will focus on the clinical utility of TMB as a predictive biomarker for ICIs efficacy in the treatment of NSCLC patients.

\section{Clinical features and prognostic value of TMB}

A retrospective analysis of 335 lung adenocarcinoma samples in a Chinese population demonstrated an association between TMB and clinical characteristics such as gender and tobacco smoking; indeed, TMB was higher in males (range 0-34 vs. 0-24 in females, $\mathrm{P}<0.001$ ) and smokers (range 0-34 vs. 0-32 in non-smokers, $\mathrm{P}=0.001$ ) (24). Alexandrov et al. also found a higher TMB in smokers compared with nonsmokers in a selection of tumors known to be associated with tobacco smoking $(\mathrm{P}<0.05)(25)$. Interestingly, subgroup analyses of NSCLC patients treated with nivolumab in second-line enrolled in the Checkmate 057 and 017 studies $(10,11)$ also found tobacco smoking to be associated with nivolumab efficacy. 
Whereas PD-L1 expression was found to be associated with tobacco smoking, TMB is not associated with high tumor cells PD-L1 expression. In a population of 240 patients with advanced NSCLC receiving anti-PD-1 or anti-PD-L1 therapies, TMB was not correlated with PD-L1 expression (Spearman $\rho=0.1915 ; \mathrm{P}=0.08$ ) (22).

However, TMB seems to be correlated with tumor genomic profile. Lower TMB has been found in tumor with $E G F R$ mutations and may explained why EGFR-mutant tumors have lower response rates to ICIs than other tumor types (26). On the contrary, KRAS and BRAF mutations are associated with higher TMB and better outcomes after ICI treatment (27).

Because high TMB induces the creation of neoantigens and is associated with intra-tumor heterogeneity, high TMB is associated with increased tumor immunogenicity and response to ICIs. For this reason, TMB is studied as a predictive biomarker of ICIs efficacy (23). In addition, TMB has previously been described as a prognostic factor for NSCLC. In a retrospective analysis of 90 patients with early-stage NSCLC who underwent surgery and received no neoadjuvant chemotherapy or immunotherapy, high TMB ( $>62)$ was associated with worse prognosis for OS $(\mathrm{HR}=7.582, \mathrm{P}=0.0018)$ and disease-free survival $(\mathrm{HR}=6.07$, $\mathrm{P}=0.0072)(28)$.

\section{Predictive value of TMB}

\section{Single agent PD-1/PD-L1 inbibitor}

\section{Pembrolizumab}

Pembrolizumab is a humanized immunoglobulin G (IgG) 4 antibody targeting PD-1. To explore the predictive value of TMB, Rizvi et al. (29) used whole-exome sequencing (WES) of NSCLC in patients with advanced disease receiving pembrolizumab. In a training cohort of 16 patients,

Patients with a durable clinical benefit (DCB; i.e., partial response or stable disease for more than 6 months) had a median number of non-synonymous mutations of 302, whereas patients with no durable benefit (NDB) had a median of 148 mutations $(\mathrm{P}=0.02)$. Seventy-three percent of patients with high TMB (defined as above the median TMB in the study population, 209) demonstrated DCB, compared with $13 \%$ of patients with low TMB $(\mathrm{P}=0.04)$. High TMB was significantly correlated with prolonged objective response rate (ORR) and PFS (ORR 63\% vs. $0 \%, \mathrm{P}=0.03$; median PFS 14.5 vs. 3.7 months, $\mathrm{P}=0.01$; HR $=0.19,95 \%$ CI: $0.05-0.70)$. In an independent validation cohort of 18 patients, patients with DCB harbored a median nonsynonymous TMB of 244 compared to 125 in patients with NDB $(\mathrm{P}=0.04)$. Patients with high TMB (defined as above the median TMB in the validation cohort) experienced higher DCB and PFS rates (DCB 83\% vs. 22\%, $\mathrm{P}=0.04$; median $\mathrm{PFS}$ not reached vs. 3.4 months, $\mathrm{P}=0.006$; $\mathrm{HR}=0.15,95 \% \mathrm{CI}: 0.04-0.59$ ). In summary, higher TMB was associated with clinical efficacy of pembrolizumab in this retrospective study with a limited number of patients (29).

\section{Nivolumab}

Nivolumab is an IgG4 fully human antibody directed against PD-1. Nivolumab was compared to platinumbased chemotherapy in an open-label phase III trial (CheckMate 026) (30). Patients with untreated stage IV or recurrent NSCLC and a PD-L1 expression $\geq 1 \%$ of tumor cells were randomized, in a 1:1 ratio, to receive either nivolumab or platinum-based chemotherapy. The primary endpoint of this trial was PFS among patients with a PD-L1 expression $\geq 5 \%$ tumor cells and was not met. Furthermore, in an exploratory analysis, no significant difference in terms of PFS was observed in the subgroup of patients with PD-L1 expression $\geq 50 \%$ tumor cells. To investigate the effect of TMB calculated by WES on patients' outcome, another exploratory analysis was conducted in 312 patients of which $58 \%$ were randomized. The patients were classified in thirds according to TMB: low TMB, between 0 and 100 mutations, medium TMB between 100 and 242 mutations, and high TMB above 243 mutations. Patients treated with nivolumab and harboring high TMB had significantly increased ORR and longer median PFS than patients treated with chemotherapy. OS was similar between groups independently of the TMB status, but $68 \%$ of patients with a high TMB in the chemotherapy group received subsequent nivolumab because of treatment crossover, access to nivolumab after the trial, or both. No significant association was observed between TMB and PD-L1 expression level (Pearson's correlation coefficient $\rho=0.059$ ). However, patients with both high TMB and a PD-L1 expression $\geq 50 \%$ tumor cells had a higher response rate $(75 \%)$ when treated with Nivolumab than patients with only one of these factors (32\% among patients with a high TMB only and $34 \%$ among those with a PD-L1 expression $\geq 50 \%$ tumor cells only) or neither factor (16\%).

CheckMate 227 was an open-label phase III trial investigating multiple hypotheses regarding the efficacy of first-line nivolumab or nivolumab-based regimens in biomarker-selected populations of advanced NSCLC 
patients (31). In the part 1 of this trial, patients with tumor expression of $\mathrm{PD}-\mathrm{L} 1 \geq 1 \%$ were randomly assigned, in a 1:1:1 ratio, to receive nivolumab plus ipilimumab (an IgG1 fully human antibody targeting CTLA-4), single agent nivolumab or platinum-based chemotherapy. Patients with tumor expression of $\mathrm{PD}-\mathrm{L} 1<1 \%$ were randomly assigned, in a 1:1:1 ratio, to receive nivolumab plus ipilimumab, nivolumab plus chemotherapy, or chemotherapy. Based on the emerging data related to TMB, the CheckMate 227 trial protocol was amended and secondary endpoints were added such as PFS with nivolumab vs. chemotherapy among patients with a $\mathrm{TMB} \geq 13$ mutation/ $\mathrm{Mb}$. TMB was calculated as the number of somatic base substitutions and short insertions and deletions (indels) per megabase of the coding analyzed genome. This TMB cutoff was retrieved from the analyses in the CheckMate 026 trial (30), including the conversion of mutation data measured by WES to mutation data obtained with the FondationOne CDx assay (31). Among 150 patients with a $\mathrm{TMB} \geq 13$ mutations/Mb and a PD-L1 expression $\geq 1 \%$ (patients with a PD-L1 expression $<1 \%$ were not eligible to receive nivolumab alone), there was no significant difference in PFS between patients treated with nivolumab as singleagent (4.2 months) and those treated with chemotherapy (5.6 months) ( $\mathrm{HR}=0.95,97.5 \%$ CI: $0.61-1.48, \mathrm{P}=0.78$ ). In conclusion, the ability of TMB to predict the efficacy of nivolumab monotherapy in patients with advanced NSCLC remains unclear.

\section{Atezolizumab}

Atezolizumab is an IgG1 humanized monoclonal antibody targeting PD-L1. Kowanetz et al. (32) explored the association between atezolizumab efficacy and TMB assessed by the FoundationOne sequencing panel (315 cancer-related genes), using pretreatment tumor specimens from 102 previously untreated and 465 previously treated NSCLC patients enrolled on three phase II atezolizumab single agent trials [POPLAR (33): randomized trial comparing atezolizumab with docetaxel in second/third line regardless PD-L1 status; BIRCH (34)/FIR (35): singlearm trials of PD-L1-selected patients]. TMB was measured using an updated TMB algorithm and the efficacy of the treatment was assessed in groups defined by the 75 th (high) and 50th (median) percentile of each study-specific TMB. In patients selected on the PD-L1 status, a higher benefit was obtained under atezolizumab when TMB was increased. In unselected patients from the POPLAR trial, the OS, PFS, and ORR benefits of atezolizumab were also prolonged in patients with increased TMB. However, TMB and PD-L1 expression were independently correlated with improved atezolizumab efficacy. In conclusion, high TMB may independently predict an improved responsiveness to atezolizumab in patients with advanced NSCLC.

Recently, Gandara et al. (36) reported the results of an exploratory study to investigate the potential interest of measuring TMB in the blood (bTMB) in the POPLAR (33) and OAK (randomized phase III trial comparing atezolizumab to docetaxel in patients with previously treated, advanced NSCLC) (37) clinical studies. The biomarker evaluable population with blood specimens available for targeted genomic sequencing included 211 patients in POPLAR and 583 patients in OAK. The bTMB assay analyzed single nucleotide variants (SNVs) in 394 genes from plasma cell-free DNA and reported a score based on the number of high-confidence SNVs detected. The results showed longer PFS and OS with atezolizumab compared to docetaxel at when bTMB levels were high. Importantly, there was no correlation between bTMB and PD-L1 expression. Therefore, the bTMB assay may be used as a non-invasive biomarker to select patients who may demonstrate clinical benefit from atezolizumab as single agent. Prospective studies investigating bTMB are currently ongoing in patients with previously untreated NSCLC (NCT02848651).

\section{Combination of PD-1/PD-L1 inbibitor and CTLA-4 inhibitor}

CheckMate 012 was an open-label, phase 1, multicohort study assessing various combinations of nivolumab plus ipilimumab in patients with previously untreated, advanced NSCLC (38). The combination of nivolumab plus ipilimumab demonstrated a tolerable safety profile and showed encouraging clinical activity in terms of response rate and duration of response. Hellmann et al. (39) retrospectively explored the correlations between TMB and treatment efficacy in 75 patients with NSCLC enrolled in the Checkmate 012 study. WES was performed on tumor tissue and paired blood, and TMB was defined as total number of nonsynonymous single nucleotide and indel variants. TMB was significantly higher in patients with an objective response (OR, complete or partial response) compared with those with no response (stable or progressive disease) (median TMB 273 vs. 114 mutations, $\mathrm{P}=0.0004$ ). Similarly, TMB was higher in patients with DCB compared 
with those with NDB (median TMB 210 vs. 113 mutations, $\mathrm{P}=0.0071)$. ORR (51\% vs. $13 \% ; \mathrm{P}=0.0005)$, $\mathrm{DCB}$ rate $(65 \%$ vs. $34 \%, \mathrm{P}=0.011)$ and $\mathrm{PFS}(\mathrm{HR}=0.41, \mathrm{P}=0.0024)$ were significantly higher in patients with high TMB (> median, 158 mutations) compared with patients with low TMB ( $\leq$ median). Interestingly, the TMB was not correlated with the PD-L1 expression. In the multivariate analysis including the PD-L1 expression, histology, smoking status, performance status and tumor burden, TMB was independently associated with ORR $(\mathrm{P}=0.001)$ and PFS $(\mathrm{P}=0.002)$. In conclusion, these data suggested that TMB could predict the efficacy of combination immunotherapy in NSCLC. However, such results had to be confirmed in an independent cohort of NSCLC patients. Moreover, a randomized trial was needed to distinguish the predictive from the prognostic value of TMB.

As previously mentioned, the CheckMate 227 trial protocol was amended to add a coprimary endpoint evaluating PFS with nivolumab plus ipilimumab versus chemotherapy among patients with a TMB $\geq 10$ mutations/ $\mathrm{Mb}$ (determined by the Fondation One CDx assay), irrespective of PD-L1 expression level (31). Of 2,877 patients enrolled in the part 1 of CheckMate 227 trial, 1,739 underwent randomization, 1,004 had valid data for TMBbased efficacy analyses, and 444 had $\geq 10$ mutations/Mb, including 139 patients treated with nivolumab plus ipilimumab and 160 patients with chemotherapy. Baseline characteristics were well balanced between the two treatment arms, notably the distribution of PD-L1 expression in tumor cells.

Among patients with high TMB, the 1-year PFS and OR rates were significantly increased with nivolumab plus ipilimumab than with chemotherapy. The duration of response (DOR) was also increased in the nivolumab plus ipilimumab arm [not reached NR (95\% CI: 12.2 months - NR)] than in the chemotherapy arm [5.4 months (95\% CI: 4.2-6.9)]. The benefit of nivolumab plus ipilimumab was consistent across subgroups, regardless of the PD-L1 expression.

By contrast, among patients with a low TMB $(<10$ mutations per $\mathrm{Mb}$ ), there was no significant difference in terms of PFS (PFS: HR $=1.07,95 \%$ CI: 0.84-1.35) between patients treated with nivolumab plus ipilimumab (median PFS $=3.2$ months, 95\% CI: 2.7-4.3) and those treated with chemotherapy (median PFS $=5.5$ months, 95\% CI: 4.3-5.6). In conclusion, these data validated the role of TMB as a potential predictive biomarker for the efficacy of the nivolumab plus ipilimumab combination in patients with advanced NSCLC.

Moreover, the Checkmate 227 trial compared the efficacy of nivolumab plus ipilimumab to nivolumab plus chemotherapy and to chemotherapy alone in patients with PD-L1 negative NSCLC. Among patients with high TMB ( 210 mutations per $\mathrm{Mb}$ ), PFS was shorter in the chemotherapy arm in comparison with the nivolumab plus chemotherapy arm (5.3 vs. 6.2 months respectively, HR $=0.56$, 95\% CI: 0.35-0.91). Among patients with low TMB ( $<10$ mutations per Mb), PFS was similar in the chemotherapy arm in comparison with the nivolumab plus chemotherapy arm (4.7 months, HR $=0.87,95 \%$ CI: 0.57-1.33).

\section{Discussion}

TMB appears to be a promising predictive biomarker to select NSCLC patients for immunotherapy, including single agent ICIs regimen or combination regimen such as nivolumab plus ipilimumab.

Concordant results were observed in small-cell lung cancer (SCLC). Hellmann et al. (40) evaluated the interest of TMB to predict the efficacy of nivolumab monotherapy or in combination with ipilimumab in 211 SCLC patients enrolled in the CheckMate 032 study. ORR was improved in patients with high TMB ( $21.3 \%$ and $46.2 \%$, respectively) than in patients with low ( $4.8 \%$ and $22.2 \%$, respectively) or medium $(6.8 \%$ and $16.0 \%$, respectively) TMB within both treatment groups. Moreover, the ORR was increased among patients treated with nivolumab plus ipilimumab than among those treated with nivolumab monotherapy within all TMB tertiles. The authors also examined the association between ORR and TMB in SCLC in comparison with the similar association in NSCLC. The receiver operating characteristics curves of the association between TMB and OR to nivolumab were comparable in patients with SCLC or NSCLC, suggesting that TMB may be used to predict the response to nivolumab in both patient populations. Furthermore, the estimated 1-year PFS rates were improved in the high TMB group $(21.2 \%$ and $30.0 \%$ for nivolumab monotherapy and nivolumab plus ipilimumab, respectively) compared with the low (not calculable and $6.2 \%$, respectively) or medium $(3.1 \%$ and $8.0 \%$, respectively) TMB groups in both patient populations. Similar trends were observed for OS. In conclusion, TMB has a conceivable usefulness as a predictive biomarker for immunotherapy efficacy throughout different types of lung cancer. 
Although TMB was historically assessed with whole genome sequencing (WGS) or whole exome sequencing (WES) (41), Rizvi et al. showed a good correlation between targeted next generation sequencing (NGS) and WES to estimate TMB (22). These results may facilitate the routine use of TMB as a predictive biomarker of ICIs efficacy. Moreover, a blood-based assay to estimate TMB would be even more convenient in routine practice (21).

TMB is a promising predictive biomarker of ICIs efficacy. High TMB indeed leads to the creation of neoantigens and increases tumor immunogenicity. However, all neoantigens do not have the same effect on tumor immunogenicity and a high intra-tumor neoantigen heterogeneity may be associated with shorter PFS with ICIs (23). For this reason, TMB still has to be validated prospectively and new biomarkers are being assessed. Neoantigen loss, defects in the interferon gamma signaling pathway or up-regulation of alternative immune checkpoints such as TIM-3, LAG-3 or TIGIT have been described as potential biomarkers of acquired resistance to ICIs (41). In addition to immune cells PD-L1 expression, other biomarkers of tumor microenvironment have been investigated such as tumor infiltrative lymphocytes (TILs) (13) or interferon signaling. In the blood, CD8 ${ }^{+}$ PD- $1^{+} \mathrm{T}$ cells expressing CD28 (42) and in the feces gut microbiome (43) also are candidate biomarkers for ICIs efficacy in NSCLC. Moreover, combinations of biomarkers may be more precise to predict ICIs efficacy in comparison with a single biomarker. This is the case for example for the combination of TMB and PD-L1 expression in comparison with TMB or PD-L1 expression alone (22). The combination of multiple biomarkers leading to the creation of a "cancer immunogram" (16) may be the optimal tool to predict ICIs potential efficacy or risk of acquires resistance in order to build a treatment algorithm for patients with advanced NSCLC.

In conclusion, more efforts have to be made to better recognize the biomarkers of benefit for unique treatment combinations. Moreover, how the sequence of different therapies may affect their benefit remains a key question. Finally, expanding this data in future clinical trials is critical for a successful development of such emerging biomarkers (41).

\section{Acknowledgements}

None.

\section{Footnote}

Conflicts of Interest: The authors have no conflicts of interest to declare.

\section{References}

1. Torre LA, Bray F, Siegel RL, et al. Global cancer statistics, 2012. CA Cancer J Clin 2015;65:87-108.

2. Mok TS, Wu YL, Thongprasert S, et al. Gefitinib or carboplatin-paclitaxel in pulmonary adenocarcinoma. N Engl J Med 2009;361:947-57.

3. Rosell R, Carcereny E, Gervais R, et al. Erlotinib versus standard chemotherapy as first-line treatment for European patients with advanced EGFR mutation-positive non-small-cell lung cancer (EURTAC): a multicentre, open-label, randomised phase 3 trial. Lancet Oncol 2012;13:239-46.

4. Sequist LV, Yang JC, Yamamoto N, et al. Phase III study of afatinib or cisplatin plus pemetrexed in patients with metastatic lung adenocarcinoma with EGFR mutations. J Clin Oncol 2013;31:3327-34.

5. Soria JC, Ohe Y, Vansteenkiste J, et al. Osimertinib in Untreated EGFR-Mutated Advanced Non-Small-Cell Lung Cancer. N Engl J Med 2018;378:113-25.

6. Solomon BJ, Mok T, Kim DW, et al. First-line crizotinib versus chemotherapy in ALK-positive lung cancer. N Engl J Med 2014;371:2167-77.

7. Soria JC, Tan DS, Chiari R, et al. First-line ceritinib versus platinum-based chemotherapy in advanced ALKrearranged non-small-cell lung cancer (ASCEND-4): a randomised, open-label, phase 3 study. Lancet 2017;389:917-29.

8. Hida T, Nokihara H, Kondo M, et al. Alectinib versus crizotinib in patients with ALK-positive non-small-cell lung cancer (J-ALEX): an open-label, randomised phase 3 trial. Lancet 2017;390:29-39.

9. Barlesi F, Mazieres J, Merlio JP, et al. Routine molecular profiling of patients with advanced non-small-cell lung cancer: results of a 1-year nationwide programme of the French Cooperative Thoracic Intergroup (IFCT). Lancet 2016;387:1415-26.

10. Brahmer J, Reckamp KL, Baas P, et al. Nivolumab versus Docetaxel in Advanced Squamous-Cell Non-Small-Cell Lung Cancer. N Engl J Med 2015;373:123-35.

11. Borghaei H, Paz-Ares L, Horn L, et al. Nivolumab versus Docetaxel in Advanced Nonsquamous Non-Small-Cell 
Lung Cancer. N Engl J Med 2015;373:1627-39.

12. Herbst RS, Baas P, Kim DW, et al. Pembrolizumab versus docetaxel for previously treated, PD-L1-positive, advanced non-small-cell lung cancer (KEYNOTE-010): a randomised controlled trial. Lancet 2016;387:1540-50.

13. Reck M, Rodríguez-Abreu D, Robinson AG, et al. Pembrolizumab versus Chemotherapy for PD-L1Positive Non-Small-Cell Lung Cancer. N Engl J Med 2016;375:1823-33.

14. Rittmeyer A, Barlesi F, Waterkamp D, et al. Atezolizumab versus docetaxel in patients with previously treated non-small-cell lung cancer (OAK): a phase 3, openlabel, multicentre randomised controlled trial. Lancet 2017;389:255-65.

15. Antonia SJ, Villegas A, Daniel D, et al. Durvalumab after Chemoradiotherapy in Stage III Non-Small-Cell Lung Cancer. N Engl J Med 2017;377:1919-29.

16. Blank CU, Haanen JB, Ribas A, Schumacher TN. Cancer Immunology. The "cancer immunogram". Science 2016;352:658-60.

17. Penault-Llorca F. Tumor mutational burden. 2017;VI:123-7.

18. Chae YK, Pan A, Davis AA, et al. Biomarkers for PD-1/ PD-L1 Blockade Therapy in Non-Small-cell Lung Cancer: Is PD-L1 Expression a Good Marker for Patient Selection? Clin Lung Cancer 2016;17:350-61.

19. McLaughlin J, Han G, Schalper KA, et al. Quantitative Assessment of the Heterogeneity of PD-L1 Expression in Non-Small-Cell Lung Cancer. JAMA Oncol 2016;2:46-54.

20. Tsao MS, Kerr KM, Kockx M, et al. PD-L1 immunohistochemistry comparability study in real-life clinical samples: results of Blueprint phase 2 project. J Thorac Oncol 2018;13:1302-11.

21. Alexandrov LB, Nik-Zainal S, Wedge DC, et al. Signatures of mutational processes in human cancer. Nature 2013;500:415-21.

22. Rizvi H, Sanchez-Vega F, La K, et al. Molecular Determinants of Response to Anti-Programmed Cell Death (PD)-1 and Anti-Programmed Death-Ligand 1 (PD-L1) Blockade in Patients With Non-Small-Cell Lung Cancer Profiled With Targeted Next-Generation Sequencing. J Clin Oncol 2018;36:633-41.

23. McGranahan N, Furness AJ, Rosenthal R, et al. Clonal neoantigens elicit $\mathrm{T}$ cell immunoreactivity and sensitivity to immune checkpoint blockade. Science 2016;351:1463-9.

24. Xiao D, Pan H, Li F, et al. Analysis of ultra-deep targeted sequencing reveals mutation burden is associated with gender and clinical outcome in lung adenocarcinoma.
Oncotarget 2016;7:22857-64.

25. Alexandrov LB, Ju YS, Haase K, et al. Mutational signatures associated with tobacco smoking in human cancer. Science 2016;354:618-22.

26. Lee CK, Man J, Lord S, et al. Checkpoint Inhibitors in Metastatic EGFR-Mutated Non-Small Cell Lung Cancer-A Meta-Analysis. J Thorac Oncol 2017;12:403-7.

27. Schrock AB, Li SD, Frampton GM, et al. Pulmonary Sarcomatoid Carcinomas Commonly Harbor Either Potentially Targetable Genomic Alterations or High Tumor Mutational Burden as Observed by Comprehensive Genomic Profiling. J Thorac Oncol 2017;12:932-42.

28. Owada-Ozaki Y, Muto S, Takagi H, et al. Prognostic Impact of Tumor Mutation Burden in Patients With Completely Resected Non-Small Cell Lung Cancer: Brief Report. J Thorac Oncol 2018;13:1217-1221.

29. Rizvi NA, Hellmann MD, Snyder A, et al. Cancer immunology. Mutational landscape determines sensitivity to PD-1 blockade in non-small cell lung cancer. Science 2015;348:124-8.

30. Carbone DP, Reck M, Paz-Ares L, et al. First-Line Nivolumab in Stage IV or Recurrent Non-Small-Cell Lung Cancer. N Engl J Med 2017;376:2415-26.

31. Hellmann MD, Ciuleanu TE, Pluzanski A, et al. Nivolumab plus Ipilimumab in Lung Cancer with a High Tumor Mutational Burden. N Engl J Med 2018;378:2093-104.

32. Kowanetz M, Zou W, Shames DS, et al. OA20.01Tumor Mutation Burden (TMB) is Associated with Improved Efficacy of Atezolizumab in 1L and 2L+ NSCLC Patients. 2017;12:S321-2.

33. Fehrenbacher L, Spira A, Ballinger M, et al. Atezolizumab versus docetaxel for patients with previously treated non-small-cell lung cancer (POPLAR): a multicentre, open-label, phase 2 randomised controlled trial. Lancet 2016;387:1837-46.

34. Peters S, Gettinger S, Johnson ML, et al. Phase II Trial of Atezolizumab As First-Line or Subsequent Therapy for Patients With Programmed Death-Ligand 1-Selected Advanced Non-Small-Cell Lung Cancer (BIRCH). J Clin Oncol 2017;35:2781-9.

35. Spigel DR, Chaft JE, Gettinger S, et al. FIR: Efficacy, Safety, and Biomarker Analysis of a Phase II Open-Label Study of Atezolizumab in PD-L1-Selected Patients with Non-Small-Cell Lung Cancer. J Thorac Oncol 2018. [Epub ahead of print].

36. Gandara DR, Kowanetz M, Mok TS, et al. 1295 O Bloodbased biomarkers for cancer immunotherapy: Tumor 
mutational burden in blood (bTMB) is associated with improved atezolizumab (atezo) efficacy in 2L+ NSCLC (POPLAR and OAK). Ann Oncol 2017;28:v460-96.

37. Rittmeyer A, Barlesi F, Waterkamp D, et al. Atezolizumab versus docetaxel in patients with previously treated non-small-cell lung cancer (OAK): a phase 3, openlabel, multicentre randomised controlled trial. Lancet 2017;389:255-65.

38. Hellmann MD, Rizvi NA, Goldman JW, et al. Nivolumab plus ipilimumab as first-line treatment for advanced nonsmall-cell lung cancer (CheckMate 012): results of an open-label, phase 1, multicohort study. Lancet Oncol 2017;18:31-41.

39. Hellmann MD, Nathanson T, Rizvi H, et al. Genomic Features of Response to Combination Immunotherapy

Cite this article as: Greillier L, Tomasini P, Barlesi F. The clinical utility of tumor mutational burden in non-small cell lung cancer. Transl Lung Cancer Res 2018;7(6):639-646. doi: 10.21037/tlcr.2018.10.08 in Patients with Advanced Non-Small-Cell Lung Cancer. Cancer Cell 2018;33:843-52.e4.

40. Hellmann MD, Callahan MK, Awad MM, et al. Tumor Mutational Burden and Efficacy of Nivolumab Monotherapy and in Combination with Ipilimumab in Small-Cell Lung Cancer. Cancer Cell 2018;33:853-61.e4.

41. Cyriac G, Gandhi L. Emerging biomarkers for immune checkpoint inhibition in lung cancer. Semin Cancer Biol 2018;52:269-277.

42. Kamphorst AO, Pillai RN, Yang S, et al. Proliferation of PD-1+ CD8 T cells in peripheral blood after PD-1targeted therapy in lung cancer patients. Proc Natl Acad Sci U S A 2017;114:4993-8.

43. Roy S, Trinchieri G. Microbiota: a key orchestrator of cancer therapy. Nat Rev Cancer 2017;17:271-85. 